\title{
Sentinel Lymph Node Biopsy in Early Breast Cancer
}

\author{
Thorsten Kühn \\ Klinik für Frauenheilkunde und Geburtshilfe, Interdisziplinäres Brustzentrum am Klinikum Esslingen, Germany
}

\section{Keywords}

Breast cancer · Surgery - Axillary lymph node dissection . Sentinel lymph node biopsy

\section{Summary}

The role of axillary surgery for the treatment of primary breast cancer is in a process of constant change. During the last decade, axillary dissection with removal of at least 10 lymph nodes (ALD) was replaced by sentinel lymph node biopsy (SLNB) as a staging procedure. Since then, the indication for SLNB rapidly expanded. Today's surgical strategies aim to minimize the rate of patients with a negative axillary status who undergo ALD. For some subgroups of patients, the indication for SLNB (e.g. multicentric disease, large tumors) or its implication for treatment planning (micrometastatic involvement, neoadjuvant chemotherapy) is being discussed. Although the indication for ALD is almost entirely restricted to patients with positive axillary lymph nodes today, the therapeutic effect of completion ALD is more and more questioned. On the other hand, the diagnostic value of ALD in node-positive patients is discussed. This article reflects today's standards in axillary surgery and discusses open issues on the diagnostic and therapeutic role of SLNB and ALD in the treatment of early breast cancer.

\section{Introduction}

Axillary surgery has always been an important component of breast cancer treatment. The goal of lymphadenectomy has changed, however, with the evolution of therapeutic concepts. In the Halstedian era, lymph node surgery was regarded as an important procedure to ensure regional control by mechanical
Schlüsselwörter

Mammakarzinom · Operation · Axilladissektion · Sentinel-Lymphknotenbiopsie

\section{Zusammenfassung}

Der Stellenwert axillärer Operationen befindet sich beim Mammakarzinom in einem kontinuierlichen Wandel. Für das axilläre Staging wurde die Axilladissektion mit Entfernung von mindestens 10 Lymphknoten (ALD) innerhalb des letzten Jahrzehnts durch die SentinelLymphknotenbiopsie (SLNB) ersetzt. Seitdem hat sich das Indikationsspektrum für die SLNB kontinuierlich erweitert. Die heutigen operativen Strategien zielen darauf $a b$, die Rate von Patientinnen zu minimieren, die trotz eines negativen Nodalstatus eine ALD erhalten. Für einige Patientinnenkollektive wird die Indikation zur SLNB (große und multizentrische Tumoren), aber auch ihre klinischen Implikationen (mikrometastatischer Befall, neoadjuvante Chemotherapie) diskutiert. Obwohl die Indikation zur ALD heute weitgehend auf nodalpositive Patientinnen beschränkt ist, wird der therapeutische Effekt der operativen Lymphknotenentfernung zunehmend hinterfragt. Andererseits wird die diagnostische Bedeutung der ALD bei nodalpositiven Patientinnen diskutiert. Dieser Beitrag stellt die heutigen Standards für die axillären Operationen dar und diskutiert die diagnostische und therapeutische Bedeutung der SLNB und der ALD für die Behandlung des frühen Mammakarzinoms.

removal of all reachable tumor cells. With the introduction of a biologic treatment approach, the lymph node status became an important criterion to determine systemic treatment decisions. The goal of axillary lymph node dissection (ALD) shifted from a purely therapeutic procedure to a diagnostic tool, which was associated with a therapeutic effect in nodepositive patients. Within the last decade, sentinel lymph node

\section{KARGER \\ Fax +497614520714 \\ Information@Karger.de}

www.karger.com

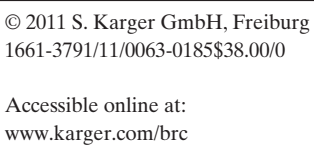

Prof. Dr. Thorsten Kühn

Städtische Kliniken Esslingen a.N.

Frauenklinik

Hirschlandstr. 97, 73730 Esslingen, Germany

Tel. +49 711 3103-3051, Fax -3052

T.Kuehn@klinikum-esslingen.de 
biopsy (SLNB) has replaced ALD as a staging procedure. SLNB is associated with considerably less morbidity without compromising staging accuracy. SLNB was primarily restricted to small and unifocal tumors. The indications have, however, rapidly expanded within the last years. Today, SLNB is indicated in practically all subgroups of patients whose lymph node status is unclear.

Although adjuvant treatment is more and more tailored by predictive factors, the axillary lymph node status is still associated with a strong prognostic power. Numerous decisions regarding the local (radiotherapy) or systemic treatment are still guided by the $\mathrm{pN}$ status. SLNB is therefore an important diagnostic tool for breast cancer treatment.

ALD is today's standard procedure to ensure regional control in patients with positive sentinel lymph nodes (SLNs). The results from earlier trials as well as recently published data question the role of ALD as a therapeutic tool, due to a high number of overtreated patients and an unfavorable therapeutic index.

The ongoing discussion on axillary surgery relates to technical standards for SLNB and the axillary management in specific situations (ductal carcinoma in situ (DCIS), multicentric disease, neadjuvant chemotherapy, etc.). The remaining role of ALD for axillary staging and its therapeutic benefit in micro- or macrometastatic lymph node involvement is intensively debated.

\section{SLNB Definition and General Remarks}

The SLN(s) is (are) defined as the first node(s) to which drainage and metastases from a primary breast cancer occurs. Intraoperatively the SLN is detected by the uptake of a tracer that is injected into the breast (tissue or skin) and imitates the lymphatic spread of tumor cells.

Numerous validation trials that performed SLNB with a backup ALD have shown that the SLN accurately stages the axilla in patients who undergo primary breast cancer surgery. A metaanalysis of more than 8,000 patients revealed a falsenegative rate (FNR) of $8 \%$ [1].

Two randomized trials compared SLNB alone with SLNB and ALD. No advantage regarding disease-free survival (DFS) and overall survival (OS) was observed for any procedure. SLNB was, however, associated with significantly less morbidity $[2,3]$.

A metaanalysis of almost 15,000 patients revealed a very low regional recurrence rate for SLNB alone of $0.3 \%$ after a median follow-up of 34 months [4].

Due to a high staging accuracy, an equivalence with ALD regarding DFS and OS (level of evidence (LOE) 1a), and significantly reduced morbidity, SLNB has replaced ALD as staging procedure to determine the $\mathrm{pN}$ status in early brast cancer.

\section{Technical Aspects}

Technical issues like the best tracer, the volume and site of injection and the use of lymphoscintigraphy (LS) have been extensively debated in the early years of SLNB. Numerous studies could show that the entire breast including the overlying skin has an identical and reproducable lymphatic drainage to the axilla, so that the injection site does not influence the FNR. Only tumors that are located close to the chest wall may have an additional drainage to the internal lymphatic bassin [5]. The injection technique is therefore influenced by technical rather than oncologic considerations. Although few comparative studies for technical variations are available, a broad consensus on the technical procedure has evolved among surgeons [6]. In a metaanalysis from 48 studies, a radiocolloid rather than a blue dye alone was applied in 47/48 studies. LS was used in 30/48 studies [4]. Although the use of LS is questioned by some surgeons, a German multiinstitutional trial showed a higher FNR in patients with less SLNs removed [7]. LS might guide the surgeon to resect all relevant SLNs. The omission of LS should therefore be based on clinical evidence.

\section{Histopathologic Examination}

The significance of low-volume metastases for further treatment decisions has not yet been clearly defined. Therefore, some variation in the extent of the histologic examination of SLNs is still existant. Most recommendations include step sectioning with intervals between 50 and $500 \mu \mathrm{m}$. A complete embedding of the SLN appears mandatory. Immunohistochemistry is not generally recommended. German guidelines recommed step sections with intervals of $500 \mu \mathrm{m}$ as a minimal standard $[8,9]$.

The intraoperative assessment of the SLNs can spare secondary axillary surgery. The most-used techniques are frozen sections and touch preparation cytology. The sensitivity of these procedures ranges between 52 and 100\% [8]. Both techniques have shown unfavorable results in micrometastatic disease. Frozen sectioning is supposed to be associated with tissue loss, which can be minimized by adequate preparation techniques.

New molecular assays using different amplification techniques for the detection of mRNA (e.g. one-step nucleic acid amplification assay, OSNA) have provided promising data for the assessment of metastatic disease in SLNs [10]. The advantage could be a reliable intraoperative detection of lymph node involvement with a reduction of secondary surgeries. The method does, however, not allow a determination of the $\mathrm{pN}$ status according to the International Union against Cancer (UICC) classification, which requires the measurement of the size of lymph node metastases. But molecular techniques might overcome the difficulties of interobserver variation in 
the interpretation of small-volume metastatic disease, if clinically relevant cut-offs can be defined.

\section{Patient Selection}

According to most published guidelines, SLNB is indicated in $\mathrm{T} 1$ and $\mathrm{T} 2$ unifocal tumors with clinically negative lymph nodes. For these tumors, an LOE 1a evidence for the equivalence with ALD has been shown in clinical trials [2, 3]. However, in their daily routine, clinicians face many individual situations that do not fit the inclusion criteria of randomized clinical trials. The level of evidence is rather low for competing options of axillary surgery. Since over- as well as undertreatment is an important surgical objective, clinical decisions must be based on existing clinical data and consensus recommendations.

\section{SLNB and Tumor Size}

Most clinical trials for the evaluation of SLNB included T1 and T2 tumors. Consecutively, most guidelines restrict SLNB to smaller tumors. A relation between tumor size and FNR has, however, never been observed [3, 7]. Available evidence and knowledge about the lymphatic drainage pattern from the breast suggest that SLNB can (and should) be performed irrespective of tumor size.

\section{Multifocal and Multicentric Carcinoma}

Numerous trials that examined the functional lymphatic drainage pattern of the breast could prove that the entire breast including the overlying skin drain through common lymphatic channels to the same axillary lymph node(s). Tumors that are located close to the chest wall may have an additional drainage to the internal lymphatic chain [5]. Several clinical trials confirmed this model, showing identical FNRs for multicentric tumors as for unifocal lesions. Patients with multifocal/ centric disease should therefore be considered as candidates for SLNB [11, 12].

\section{SLNB and Clinically Suspicious Nodes}

Most guidelines recommend SLNB in patients with a clinically negative axillary status. The term 'clinically negative' is, however, insufficiently defined. Specht et al. [13] could show that the clinical examination of the axilla was falsely positive in $53 \%$ of patients with moderately suspicious nodes and in $23 \%$ of patients with highly suspicious nodes. In order to reduce unnecessary overtreatment, a new algorithm for the preoperative evaluation of the axilla is being recommended [14]:
Axillary ultrasound is mandatory prior to any lymph node surgery for breast cancer. In cases of suspicious nodes, a fineneedle aspiration (FNA) or a core needle biopsy (CNB) should be performed.

\section{Prior Breast Surgery}

The data about the influence of a prior excisional biopsy on the FNR are contradictory. In the largest randomized trial [3], patients with a previous breast operation had a significantly worse FNR compared to patients who underwent primary breast and axillary surgery. There is, however, no information on the resection volume, which probably influences the drainage pattern for secondary lymphatic mapping. Prior excisional biopsy is not considered a contraindication for SLNB.

\section{SLNB in Pregnant Women}

A study from the Memorial Sloan-Kettering Cancer Center could show that the SLN procedure with a radioactive tracer leads to a negligible dose to the fetus [15]. The organ and fetal absorbed dose estimates are well below $50 \mathrm{mGy}$, which is believed to be the threshhold absorbed dose for adverse effects and much lower than the US National Council on Radiation Protection and Measurements limit to a pregnant woman. Pregnancy should therefore not be regarded as a contraindication for SLNB.

\section{SLNB in the Neoadjuvant Setting}

Neoadjuvant chemotherapy (NCHT) is increasingly being offered to patients with early-stage breast cancer to improve their eligibility for breast concervation therapy (BCT). The introduction of SLNB has challenged the classical standard of ALD in the setting of NCHT. Up to $70 \%$ of the women have uninvolved lymph nodes after chemotherapy, and $20 \%$ of them convert from a positive to a negative axillary status [16, 17]. SLNB after NCHT could spare many patients from unnecessary ALD. Although numerous studies indicate similar FNRs after NCHT compared to primary surgery, all of these studies are unicentric trials with a retrospective design that include relatively few patients [18]. The results regarding the feasiblity (detection rate) and the reliability of SLNB to predict the axillary status (FNR) are heterogeneous.

The axillary status remains a strong prognostic factor that tailors many adjuvant treatment decisons (systemic treatment, radiotherapy). This relates, however, to the $\mathrm{pN}$ status prior to treatment, while the significance of the ypN status for clinical decision making is unclear. 
The role of SLNB (prior to or after NCHT) remains controversial. The German SENTINA (SENTInel NeoAdjuvant) trial of the AGO-B (Arbeitsgemeinschaft gynäkologische Onkologie) is a prospective multicenter study that examines the role of SLNB in the neodjuvant setting [17]. More than 1500 patients will be included. This trial will provide important information for the improvement of targeted axillary surgery in patients who undergo NCHT.

\section{SLNB and DCIS}

DCIS is a preinvasive lesion. Axillary staging is therefore not required as a standard procedure. Axillary involvement due to the presence of unrecognized invasive disease is less than $5 \%$. SLNB is recommended as a second procedure in patients with unexpected proof of invasive carcinoma [18]. Primary SLNB should be considered in patients whose lymphatic drainage pathways to the axilla are destroyed by the excision of the primary lesion, either due to a large resection volume or a close relation of the lesion to the axillary tail (e.g. mastectomy, large resection specimen). In BCT, the decision for a primary SLNB must be taken on an individual basis. German guidelines recommend SLNB in patients who undergo mastectomy for extensive DCIS [8, 9].

\section{Re-SLNB in Patients with Local Recurrence after BCT and SLNB}

About $5-10 \%$ of patients who underwent BCT and SLNB will develop local recurrence in the breast. The management of the axilla for these patients is still not defined. Fehm et al. [19] reviewed the literature on the role of SLNB in the reoperative setting. The detection rate ranged between 55 and 97\%. Remarkably, the rate of extraaxillary SLNs was as high as $8-51 \%$. Up to $35 \%$ of the patients had tumor-positive nodes.

It appears that the breast develops new lymphatic drainage patterns after damage to the primary lymphatic system caused by therapy. The topography of this new drainage is significantly more variable than in an untreated breast. The exact mechanisms and time needed to develop a new drainage system is unclear.

Since SLNB is primarily a staging procedure that tailors adjuvant therapy, the clinical implications of a re-operative SLNB regarding the systemic treatment are not defined. Due to a high rate of extraaxillary SLN involvement, the role of further local treatment is equally difficult to decide. Due to the undefined clinical consequences, a re-operative SLNB cannot be recommended as a standard procedure. Further trials should elucidate the role of re-operative SLNB.

\section{Intramammary SLN}

Intramammary lymph nodes (IMLs) are defined as lymph nodes that are surrounded by glandular tissue. The prevalence of IMLs has been reported to range between 1 and 28\%. IML metastases are an independent predictor of poor outcome [20]. Patients with positive IMLs are staged as pN1. Egan and McSweeney [21] could not show a relation of IMLs to the common lymphatic pathways of the breast. The IMLs did not communicate with the subcutaneous valveless vessels that play an important role in the lymphatic spread of breast cancer cells. In analogy to melanoma, IMLs can therefore be regarded as 'transient nodes'. ALD can be omitted in patients with a positive IML if an axillary SLN is identified and yields a negative result. Patients with IML involvement who have negative axillary lymph nodes should receive systemic treatment according to node-positive patients.

\section{Extraaxillary SLNB}

So far, there is no evidence that extraaxillary SLNB is associated with improved DFS or OS. On the other hand, the procedure leads to additional surgical risks. Extraaxillary SLNB is not recommended in routine clinical practice [22].

\section{SLNB and Low-Volume Metastatic Involvement - Implication for Systemic Treatment Decisions}

SLNB provides the opportunity for a more detailed analysis of the lymph node status including serial sections and immunohistochemistry of 1 or a few SLNs. The clinical significance of a low tumor burden in the SLN regarding prognosis and consecutive systemic treatment is not yet fully understood.

The prognostic role of 'occult' axillary lymph node metastases has been discussed for a long time with contradictory results. The comparison of earlier studies was compromised by the lack of a clear definition of the term 'occult'. The UICC (6th edition) provided a metric classification for minimal lymph node involvement: tumor deposits $\leq 0.2 \mathrm{~mm}$ are defined es pN0(i+) (isolated tumor cells), while metastases $>0.2 \mathrm{~mm}$ and $\leq 2 \mathrm{~mm}$ are categorized as $\mathrm{pN1}$ (mi) (micrometastasis) [23]. Most recently, several studies on the significance of low-volume metastases have been published that define a minimal lymphatic tumor burden according to the UICC staging system. These studies provide valuable new data for a better guidance of clincal treatment decisions:

Tan et al. [24] could show in a retrospective analysis of 368 patients who received no adjuvant systemic treatment that the number of metastatic cells and the cluster size were significantly related to DFS and OS after a follow-up of 20 years.

The Dutch MIRROR (Micrometastases and Isolated Tumor Cells: Relevant and Robust or Rubbish?) study, a 
retrospective cohort study with a total of 2707 patients with or without micrometastatic involvement who received either systemic treatment or not, demonstrated that isolated tumor cells or micrometastases in regional nodes were associated with a reduced 5-year DFS in women who did not receive adjuvant therapy. Systemic treatment improved DFS in these patients [25].

The National Surgical Adjuvant Breast and Bowel Project (NSABP) B-32 study was initially designed to evaluate the equivalence of SLNB with SLNB and ALD. At the San Antonio Breast Cancer Symposium 2010, the prospective outcomes of patients with micro- and macrometastases were presented. Almost $85 \%$ of the patients had received systemic therapy. A significant difference between node-positive and nodenegative patients was observed with respect to DFS and OS. In contrast, no difference was found between node-negative patients and those with micrometastatic involvement [26].

The following conclusions can be drawn from these three newly published trials that defined micrometastatic lymph node involvement according to the current UICC classification:

Micrometastatic involvement is associated with a worse DFS and OS only in the absence of systemic treatment. Patients who receive any systemic medication have an identical prognosis to node-negative patients. Since today's guidelines recommend a systemic treatment in practically all patients, these data confirm former suggestions that, in contrast to macrometastatic disease, exclusive micrometastatic lymph node involvement should not influence the kind of systemic treatment decision [7].

\section{SLNB and Low-Volume Metastatic Involvement - Implications for ALD}

Between $0-57 \%$ of non-SLN involvement has been reported in women with micrometastatic disease in the SLN [27]. The role of ALD for regional control in these patients is unclear. ALD may detect additional nodal involvement that upstages the $\mathrm{pN}$ status and would influence systemic treatment.

The Dutch MIRROR study is the largest retrospective cohort study that examined the impact of omission of axillary treatment (ALD or radiotherapy) on the regional recurrence rate in a total of $835 \mathrm{pN} 0(\mathrm{i}-), 799 \mathrm{pN} 0(\mathrm{i}+)$ and $958 \mathrm{pN} 1(\mathrm{mi})$ patients. Omission of any axillary treatment resulted in a higher 5-year recurrence rate in $\mathrm{pN} 1(\mathrm{mi})$ but not in $\mathrm{pN} 0(\mathrm{i}+)$ patients (5\% vs. $1 \%$, hazard ratio (HR) 4.39) [28], even after correction for adjuvant systemic treatment.

No specific data on the effect of ALD as a staging procedure (upstaging from $\mathrm{pN} 0(\mathrm{i}+), \mathrm{pN} 1(\mathrm{mi})$ to $\mathrm{pN} 1$ ) on DFS and OS (due to a modified systemic treatent) are available. Patients with a pN1 status would derive a significant benefit from chemotherapy and the use of taxanes [29, 30]. Since the rate of non-SLN involvement is quite high in some studies $(0-57 \%)$, the role of ALD as a staging procedure in patients with micrometastatic SLN involvement requires further evaluation.

The International Breast Cancer Study Group (IBCSG) 23-01 trial, which randomizes patients with micrometastases in the SLN to ALD versus no further axillary treatment, will probably elucidate the issue of regional treatment in patients with micrometastatic SLN involvement. Until then, the decision for ALD in patients with a $\mathrm{pN} 1$ (mi) status should be individualized. In women who receive $\mathrm{BCT}$ and tangential field irrradiation, the effect of ALD on regional control appears minimal. If the systemic treatment decision will not be modified by an eventual alteration of the $\mathrm{pN}$ status, omission of ALD appears permissable.

\section{SLNB and Macroscopic Metastatic Involvement - Implications for Further Axillary Treatment}

ALD has been the standard treatment for SLN-positive patients during the last decade. Most recently, the role of ALD for these patients has increasingly been questioned. At the American Society of Clinical Oncology (ASCO) meeting 2010, the American College of Surgeons Oncology Group (ACOSOG) reported data from a randomized trial (Z11) that compared ALD versus no specific axillary treatment among 856 patients [31]. The 5-year regional recurrence rate was $0.9 \%$ for SLNB compared to $0.5 \%$ for ALD ( $\mathrm{p}=0.11)$, with 5 -year OS rates of 92.5 and $91.9 \%$, respectively $(\mathrm{p}=0.24)$. Of note, $27 \%$ of the patients in the ALD group had further nonSLN involvement. Some critical issues relating to this study require further discussion:

The Z11 trial did not reach the targeted accrual. All patients received BCT with tangential field irradiation of the remaining breast. This treatment includes an irradiation of the lower axilla. Furthermore, the tumor characteristics in the study were rather favorable. Micrometastatic SLN involvement was found in $37.5 \%$ of patients in the ALD group and $44.8 \%$ of the SLN group. The low locoregional recurrence rate of $3.4 \%$ at 5 years confirms the rather favorable patient characteristics in the study.

In contrast to $\mathrm{Z} 11$, there are some data in the literature that suggest an impact of axillary surgery on OS. In a Bayesian metaanalysis, the combination of trials including almost 3000 patients showed a $5.4 \%$ survival benefit in favor of ALD [32]. In addition, 6 large pre-SN database studies, including a total of 160,459 patients, showed a survival benefit in direct proportion to the number of lymph nodes removed [33]. The largest single-center study reported data from 287 patients who received no further axillary treatment, in patients with a positive SLN. Although $15 \%$ of the patients received radiotherapy to the axilla and/or supraclavicular nodes, axillary recurrences developed in up to $5.0 \%$ of the patients at a median follow-up of only 23 months [34]. The MIRROR study found 
$5.0 \%$ regional recurrences in patients with micrometastatic SLN involvement if the axilla was left untreated. These axillary recurrence rates are much higher than those decribed in $\mathrm{Z} 11$.

Although Z11 questions the role of surgery for SLN-positive patients, the omission of ALD can not yet be declared as a new standard of care, especially as the explanation for the low regional recurrence rate is unclear [35]. The results may be explaned by a therapeutic effect of radiotherapy. On the other hand, there may be a biological explanation that is not yet well understood. The NSABP 04 trial randomized clinically negative patients to radical mastectomy, total mastectomy with postoperative irradiation, and total mastectomy alone without axillary treatment. $38 \%$ of the women whose axillae were dissected had tumor-involved nodes. In the group of patients whose axillae were left untreated, the rate of clinically apparent nodal disease was less than half [36]. Z11 might confirm the observation that tumor-involved nodes do not necessarily become clinically apparent. The biological reason for this is unkown.

It can be concluded from Z11 and other trials that the therapeutic effect of ALD on regional tumor control appears small and that the therapeutic index seems unfavorable due to a high surgery-related morbidity. There is, however, insufficient evidence to suggest the omission of axillary treatment in all patients with a positive SLN. This relates especially to patients who undergo mastectomy without irradiation of the thoracic wall and to patients with partial breast irradiation (intraoperative radiotherapy, IORT).

Another issue is the diagostic effect of ALD in patients with a positive SLN. There is some evidence that patients with a low axillary tumor burden (1-3 nodes) have the greatest benefit from a taxane-containing chemotherapy, whereas patients with a higher lymph node involvement may require more intensive systemic treatment [37]. About $10 \%$ of the patients in Z11 had more than 4 involved nodes. It is unclear if the systemic treatment for these patients was different compared to the others. A possible difference in outcome would have no statistical impact on DFS and OS of the entire study due to the low number of patients.
The AMAROS (After Mapping of the Axilla: Radiotherapy or Surgery?) trial of the European Organisation for Research and Treatment of Cancer (EORTC) compares ALD with radiotherapy of the axilla in SLN-positive patients. The results of this large randomized trial should be awaited before treatment algorithms are changed. If ALD is omitted in selected cases, these patients should fulfil exactly the inclusion criteria of Z11. Radiotherapy should be regarded as an option, especially if no further lymph node involvement is expected. Nomograms as mathematical models to predict the likelihood of further lymph node involvement may be a helpful tool to select patients who are unlikely to be upstaged by further axillary surgery.

\section{Conclusions}

SLNB has evolved as the new standard of care for determining the $\mathrm{pN}$ status in early breast cancer. The indication for SLNB has rapidly expanded. Primary ALD for clinically suspicious nodes should be based on a cytological or histological examination from FNA or CNB. The role of SLNB in the neoadjuvant setting needs to be determined. Completion axillary surgery (ALD) in SLN-positive patients is increasingly questioned since the therapeutic index appears unfavorable. The new recommendations of the German AGO downstaged the recommendation for ALD in patients who fulfill the inclusion criteria of Z11 (T1-2 tumors) who receive BCT and whole breast irradiation. There are, however, insufficient data to recommend omission of axillary treatment (ALD or radiotherapy) in SLN-positive patients. The role of ALD as staging procedure in node-positive patients remains a matter of debate.

\section{Disclosure Statement}

There have been no financial interests/arrangements with one or more organisations that could be perceived as a real or apparent conflict of interest in the context of the subject of this article.

\section{References}

1 Kim T, Giuliano AE, Lyman GH: Lymphatic mapping and sentinel lymph node biopsy in early stage breast carcinoma: a metaanalysis. Cancer 2006;106:4-16.

2 Veronesi U, Viale G, Paganelli G, et al.: Sentinel lymph node biopsy in breast cancer: ten-year results of a randomized controlled trial. Ann Surg 2010;251:595-600.

3 Krag DN, Anderson SJ, Julian TB, et al.: Sentinellymph-node resection compared with conventional axillary lymph node dissection in clinically nodenegative patients with breast cancer: overall survival findings from the NSABP B-32 randomised phase 3 trial. Lancet Oncol 2010;11:927-933.
4 van der Ploeg IM, Nieweg OE, van Rijk MC, Valdes Olmos RA, Kroon BB: Axillary recurrence after a tumour-negative sentinel node biopsy in breast cancer patients: A systematic review and meta-analysis of the literature. Eur Surg Oncol 2008;34:1277-1284.

5 Roumen RM, Geuskens LM, Valkenburg JG: In search of the true sentinel node by different injection techniques in breast cancer patients. Eur J Surg Oncol 2000;26:195-196.

6 Kühn T: Clinical aspects of sentinel lymph node biopsy (Expert Discussion). Breast Care 2007; 2:234-239

\footnotetext{
7 Kuehn T, Vogl FD, Helms G, v Pueckler S, Schirrmeister H, Strueber R, Koretz K, Kreienberg R: Sentinel-node-biopsy is a reliable method for axillary staging in breast cancer: results from a large prospective German multi-institutional trial. Eur J Surg Oncol 2004;30:252-259.

8 Kuehn T, Bembenek A, Decker T, Munz DL, Sautter-Bihl ML, Untch M, Wallwiener D Consensus Committee of the German Society of Senology: A concept for the clinical implementation of sentinel lymph node biopsy in patients with breast carcinoma with special regard to quality assurance. Cancer 2005;103:451-461.

9 www.ago-online.de.
} 
10 Rubio IT, Aznar F, Lirola J, Peg V, Xercavins J: Intraoperative assessment of sentinel lymph nodes after neoadjuvant chemotherapy in patients with breast cancer. Ann Surg Oncol 2010;17:235-239.

11 Knauer M, Konstantniuk P, Haid A, et al.: Multicentric breast cancer: a new indication for sentinel-node-biopsy - a multicentric validation study. J Clin Oncol 2006;24:3374-3380.

12 Schrenk P, Wayand W: Sentinel-node biopsy in axillary lymph-node staging for patients with multicentric breast cancer. Lancet 2001;357:122.

$\checkmark 13$ Specht MC, Fey JV, Borgen PI, Cody HS III: Is the clinically positive axilla in breast cancer really a contraindication to sentinel lymph node biopsy? J Am Coll Surg 2005;200:10-14.

14 Gruber I, Hahn M, Fehm T, v Weyhern $\mathrm{CH}$, Stäbler A, Winckelmann A, Wallwiener D, Kühn T: Relevance and methods of interventional breast sonography in preoperative axillary lymph node staging. Eur J Ultrasound 2011, in press.

15 Pandit-Tasker N, Dauer LT, Montgomery L, St Germain J, Zanzonico PB, Divgi CR: Organ and fetal absorbed dose estimates from 99mTc-sulfur colloid lymphoscintigraphy and sentinel node localisation in breast cancer patients. J Nucl Med 2006;47:1202-1208.

16 Untch M, Fasching PA, Konecny GE, et al.: Pathological complete response after neoadjuvant chemotherapy + trastuzumab treatment predicts survival. Fourty one month median follow-up data of the multicenter TECHNO trial. J Clin Oncol 2011, in press.

17 Kuerer HM, Sahin AA, Hunt KK, et al.: Incidence and impact of documented eradication of breas cancer axillary lymph node metastases before surgery in patients treated with neoadjuvant chemotherapy. Ann Surg 1999;230:72-78.

-18 Xing Y, Foy M, Cox DD, et al.: Meta-analysis of sentinel lymph node biopsy after preoperative chemotherapy in patients with breast cancer. Br J Surg 2006;93:539-546.

19 Fehm T, Helms G, Banys M, Kuehn T: Management of the axilla for ipsilateral breast cancer recurrence after previous sentinel lymph node biopsy and breast conserving therapy. Gynecol Surg 2011;8:13-18.
20 Nassar A, Cohen C, Cotsonis G, Carlson G: Significance of intramammary lymph nodes in the staging of breast cancer: correlation with tumor characteristics and outcome. Breast 2008;14:147-152.

21 Egan RL, McSweeney MB: Intramammary lymph nodes. Cancer 1983;51:1838-1842.

22 Cody HS 3rd: Clinical significance and management of extra-axillary sentinel lymph nodes: worthwhile or irrelevant? Surg Oncol Clin N Am 2010;19:507-517.

23 Green FL, Page DL, Fleming ID, et al. (eds): AJCC Cancer Staging Manual, ed 6. Chicago, American Joint Commission on Cancer, 2002.

24 Tan LK, Giri D, Hummer AJ, Panageas KS, Brogi E, Norton L, Hudis C, Borgen PI, Cody HS: Occult axillary node metastases in breast cancer are prognostically significant: results in 368 nodenegative patients with 20 -year follow-up. J Clin Oncol 2008;26:1803-1809.

25 De Boer M, van Deurzen C, van Dijck J, Borm GF, van Diest PJ, Adang EM, Nortier J, Rutgers EJT, Seynaeve C, Menke-Pluymers M, Bult P, TjanHeijnen VCG: Micrometastases or isolated tumor cells and the outcome of breast cancer. N Engl J Med 2009;361:653-663.

26 Julian TB, Anderson SJ, Golesorkhi N, Fourchette V, Mamounas EP, Wolmark N: Prospective outcomes for patients with micrometastases and macrometastases in sentinel nodes: NSABP B-32 sentinel node trial. SABCS 2010; abstr S-1.

27 Cserni G, Gregori D, Merletti F, et al.: Metaanalysis of non-sentinel node metastases associated with micrometastatic sentinel nodes in breast cancer. $\mathrm{Br}$ J Surg 2004;91:1245-1252.

28 Tjan-Heijnen VC, Pepels MJ, de Boer M, et al.: Impact of omission of completion axillary lymph node dissection or axillary therapy in breast cancer patients with micrometastases $(\mathrm{pN} 1 \mathrm{mi})$ or isolated tumor cells $(\mathrm{pNO}(\mathrm{i}+))$ in the sentinel lymph node (SN): results from the MIRROR study. Orlando, American Society of Clinical Oncology, 2009, CRA506.

29 Martin M, Pienkowski T, Mackey J: Adjuvant docetaxel for node positive breast cancer. N Engl J Med 2005;352:2302-2313.
30 Roche H, Fumoleau P, Spielmann M, et al.: Sequential adjuvant epirubicin-based and docetaxel chemotherapy for node positive breast cancer patients: the FNCLCC PACS 01 trial. J Clin Oncol 2006;24:5664-5671.

31 Giuliano AE, McCall L, Beitsch P, Whithworth PW Blumencranz P, Leitch AM, Saha S, Hunt KK, Morrow M, Ballman K: Locoregional recurrence after sentinel lymph node dissection with or without axillary dissection in patients with sentinel lymph node metastases. Ann Surg 2010;252:426433.

32 Orr RK: The impact of prophylactic axillary node dissection on breast cancer survival - a Bayesian meta-analysis. Ann Surg 1999;6:109-116.

33 Krag DN, Single RM: Breast cancer survival according to number of nodes removed. Ann Surg Oncol 2003;10:1152-1159.

34 Park J, Fey JV, Naik AM, et al.: A declining rate of completion axillary dissection in sentinel lymph node positive breast cancer patients is associated with the use of a multivariate nomogram. Ann Surg 2007;245:462-468.

35 Kühn T: The role of axillary lymph node dissection in SLN positive patients - are the results of Z11 practice changing? Breast Care 2010;5:431-432

36 Fisher B, Jeong JH, Anderson S, et al.: Twentyfive-year follow-up of a randomized trial comparing radical mastectomy, total mastectomy and total mastectomy followed by irradiation. N Engl J Med 2002;347:567-575.

37 Moebus V, Jackisch C, Luecvk HJ, et al.: Intense dose-dense sequential chemotherapy with epirubicin, paclitaxel and cyclophosphamide compared with conventionally scheduled chemotherapy in high risk primary breast cancer: mature results of an AGO phase III study. J Clin Oncol 2010;28:2874-2880. 